Original article

\title{
The thyroid eye disease (TED) laterality debate: A comparison of characteristics, presentation, severity, and activity, between unilateral and bilateral thyroid eye disease
}

\author{
Ronald Steven S. Medalle II, MD, MBA ${ }^{\mathrm{a}}$, Red Thaddeus D. Miguel, MD, MBA ${ }^{\mathrm{b}, *}$ \\ ${ }^{a}$ Department of Health Eye Center - East Avenue Medical Center, Philippines \\ ${ }^{\mathrm{b}}$ Asia-Pacific Center for Evidence-Based Healthcare, Manila, Philippines
}

\section{A R T I C L E I N F O}

\section{Keywords:}

Thyroid eye disease

Graves

Endocrinology

Ophthalmology

Clinical

\begin{abstract}
A B S T R A C T
Background/objectives: Thyroid eye disease (TED), which can lead to severe sequelae, can involve either one eye or both eyes. The importance of knowing the distinction between laterality rests on the risk that either presentation is associated with a more severe form. It is our aim to investigate differences in patient characteristics and presentations between unilateral and bilateral TED.

Methods: Cross-sectional study on clinically diagnosed TED patients from December 2013 to April 2018. Patients' sociodemographic factors, medical history, presentation, severity, and activity between unilateral TED and bilateral TED were compared.

Results: 65 patients were included (unilateral TED: $\mathrm{n}=40,61.5 \%$; bilateral TED: $\mathrm{n}=25,38.5 \%$ ). Unilateral and bilateral TED were not different with regards to age, gender, family history of thyroid disease, comorbidities, and smoking status. There was nearly six times the likelihood of methimazole intake being associated with bilateral TED (odds ratio $[\mathrm{OR}]=5.80, \mathrm{p}=0.02$ ). Both groups were similar in almost all general presentation, orbital inflammation signs, and manifestations of lid retraction. The exception being blurred vision that was more common among bilateral TED patients (OR $=4.80, \mathrm{p}=0.04)$. There were also no differences between both groups in terms of thyroid hormones (TSH: $\mathrm{p}=0.84$; freeT4: $\mathrm{p}=0.12)$, severity $(\mathrm{p}=0.61)$, and activity $(\mathrm{p}=$ 0.99).

Conclusions: Our study found unilateral TED to be more prevalent, while ongoing methimazole treatment and blurred vision are associated with bilateral TED. Our findings add to the growing evidence suggesting that the laterality of TED is not a factor in differentiating levels of activity or severity.
\end{abstract}

\section{Introduction}

Thyroid eye disease (TED) is the most common extrathyroidal involvement of Graves' disease while also being present among euthyroid or hypothyroid patients. ${ }^{1-3}$ TED presents with visible signs in half of Graves' disease patients, which can manifest as eyelid retraction to more severe and sight-threatening sequelae. ${ }^{4,5}$ Although not completely understood, the pathophysiology of TED has been increasingly linked to sensitised $\mathrm{T}$ lymphocytes and autoantibodies directed against antigens common between the orbit and the thyroid. ${ }^{6-8}$

TED can involve either one eye (unilateral TED), or both eyes (bilateral TED). Many theories surround the laterality of TED, with some suggesting a progression from unilateral TED to bilateral TED. ${ }^{9}$ Other researchers on the other hand suggest that patients from the beginning have both eyes involved in the disease process, yet symptoms are only observable unilaterally in some patients. ${ }^{10}$ Given the differences in the possible explanations for either types of TED, the importance of knowing the distinction between laterality lies in the possible risk that either of the presentations may be associated with a more severe form of the disease.

An earlier study published by Kashkouli and colleagues investigated whether there was in fact a possibility that either laterality types was related to a more severe and active form of TED. ${ }^{11}$ From 851 patients, 303 of which had TED (32 with unilateral TED; 10.6\%), the study found that laterality of TED was not associated with either a more severe form of TED or active TED. ${ }^{11}$

\footnotetext{
* Corresponding author.

E-mail addresses: stevenmedalle@gmail.com (R.S.S. Medalle II), rtdmiguel@gmail.com (R.T.D. Miguel).
} 
Stemming from the study by Kashkouli and colleagues it was our aim to investigate differences in sociodemographic factors, medical history, presentation, severity of TED, and rates of TED activity between unilateral TED and bilateral TED in the Philippines. Furthermore, the study by Kashkouli and colleagues was completed in 2006. Since completion and corresponding dissemination, a vast number of studies have since been published. ${ }^{12}$ Many physicians are likely more aware of the complexities in TED's presentation and may reflect a difference in stage of diagnosis and rate of diagnosis. We therefore attempt to test the consistency of findings of the study by Kashkouli and colleagues, years after the publication of the original paper.

\section{Methods}

The study drew respondents from a sample of all clinically diagnosed TED patients from December 2013 to April 2018 at a specialized eye health facility in Quezon City, Philippines. The cross-sectional design collected data from patient records. The study was approved by the facility's ethics review board.

Patients' charts were retrieved using the annual census where identification numbers were tagged as having been diagnosed with TED. Only records from the initial visit were used in the study to avoid confounding due to variations in treatment. Patients previously diagnosed with TED from another institution were excluded from the study. The sample size was estimated to be at 58 patients to estimate an active TED rate of $8 \%$ and a moderate to severe TED rate of $8 \% .{ }^{13}$ Computations for power was computed with a margin of error of $7 \%$ and an alpha of 0.05 .

\subsection{Measures}

The main outcome of interest was laterality of TED. The diagnosis of TED at the institution is based on the criteria by Bartley and Gorman (1995). ${ }^{14}$ Patients were classified as unilateral TED when the record indicated a final diagnosis involving only one eye. A diagnosis of TED indicating an involvement of both eyes led to a classification of bilateral TED. Further, it was decided a priori that despite the presence of clinical findings or imaging results in the patient's charts, which may be used to construct a diagnosis, the final diagnosis of the attending physician at the time of visit was upheld as the basis for TED laterality classification. This was decided to avoid possible misinterpretation of results on other sections of the patient's charts due to possible missing data, errors in encoding of the information in the charts, or other clerical factors that would have inhibited us from understanding the full scope of the patient's condition.

Socio-demographic information, family and medical history, laboratory levels of thyroid stimulating hormone (TSH) and free thyroxine (T4), presenting symptoms, and clinical manifestations of TED were collected and compared between unilateral and bilateral TED.

Socio-demographic information included sex and age, where the latter was recorded as a continuous variable. Family and medical history comprised of family history of thyroid disease, current treatment with anti-thyroid medications (ATD), and smoking history. Smoking history was noted as either currently smoking, previous smoker (previous cessation prior to visit), or never smoked cigarettes. Thyroid hormones were recorded when available. Observations from ophthalmologic history and examination was also recorded and compared by laterality of TED.

The Clinical Activity Score (CAS) system by Mourits and colleagues was used to assess inflammatory process of TED in patients (i.e., TED activity). ${ }^{15}$ The parameters included spontaneous orbital pain, gaze evoked orbital pain, eyelid swelling, eyelid erythema, conjunctival redness, chemosis, and inflammation of caruncle or plica. A score of three or more points out of seven indicated active TED.

The European Group of Graves' Orbitopathy (EUGOGO) classification was used to describe the risk of vision loss in a patient. ${ }^{16}$ Mild ophthalmopathy was classified in a patient with one or more of the following signs: minor lid retraction $(<2 \mathrm{~mm})$, mild soft tissue involvement, exophthalmos $(<3 \mathrm{~mm})$, transient or no diplopia, and corneal exposure responsive to lubricants; Moderate to severe ophthalmopathy was present with one or more of the following signs: lid retraction $(>2 \mathrm{~mm})$, moderate to severe tissue involvement, exophthalmos $(>3 \mathrm{~mm})$, and with inconstant, or constant diplopia. Lastly, Sight-threatening Ophthalmopathy was considered when ocular globe subluxation, severe forms of frozen eye, choroidal folds, or postural visual darkening was present or if the patient presented with dysthyroid optic neuropathy or corneal breakdown.

Other measurements available included lid retraction, measured by margin reflex distance, and proptosis, measured using a Hertel exophthalmometer. Optic nerve involvement was determined in the presence of a relative afferent pupillary defect.

\subsection{Data analysis}

Data was checked for completeness and encoded in Microsoft Excel 2019. Descriptive statistics including proportion and means was used to describe the data. Fisher's exact test was used to compare categorical variables between unilateral TED and bilateral TED. Continuous variables were analyzed using student's t-test when the data was normally distributed and the Mann-Whitney $U$ test when the data was not normally distributed. Normality testing was performed using the Shapiro-Wilk test. A logistic regression model examined the association between variables that were significantly different between TED laterality groups. Odds ratio (OR) and 95\% confidence interval (CI) declared the independent effect of variables on the outcome. An alpha level of 0.05 were considered statistically significant. Interferential analysis was carried out using R 3.5.1.

\section{Results}

From December 2013 to April 2018 the database identified 65 patients that met the inclusion criteria for the study. Thorough validation of individual patient records revealed that all patient records initially screened had all fundamental information present and all were deemed eligible. Among the 65 patients, $40(61.5 \%)$ were noted to have unilateral TED and 25 (38.5\%) were noted to have bilateral TED.

Patients with unilateral TED were slightly older with a mean age of 39 years old (SD:15.2), compared to patients with bilateral TED. Further, unlike the bilateral TED group, unilateral TED patients were predominantly female $(70 \%$ female). The differences in the age and gender distributions between both groups, however, were not statistically significant. Similarly, both unilateral and bilateral TED groups were not different when variables of family history of TED, comorbidities, and smoking status were examined (Table 1).

Both groups were only statistically different when rates of ATD were compared between unilateral and bilateral TED patients. Specifically, the analysis found that current methimazole intake was more common among bilateral TED patients than unilateral TED patients $(\mathrm{p}=0.02)$. Results of regression analysis yield an increased likelihood of current methimazole intake (ref: no methimazole intake) being associated with bilateral TED compared to unilateral TED (odds ratio [OR] $=5.80,95 \%$ CI: $1.37-24.64 ; \mathrm{p}=0.02$ ).

There was no statistically apparent difference between unilateral and bilateral TED patients when signs and symptoms were investigated between both groups; except for blurred vision that was more common among bilateral TED patients $(\mathrm{p}=0.0365)$ (Table 2$)$. Odds ratio for blurred vision (reference: no blurred vision) being associated with bilateral TED compared to unilateral TED was 4.80 (95\%CI: 1.11-20.76; $\mathrm{p}=0.04$ ).

Major thyroid hormones were available for 41 patients (unilateral $n$ $=29$, bilateral $\mathrm{n}=12$ ). TSH was similar in bilateral TED (mean TSH $=$ $2.13 \mathrm{mU} / \mathrm{L}$ [SD:3.1]) and unilateral TED (mean TSH $=2.13 \mathrm{mU} / \mathrm{L}$ [SD:3.1]), $(\mathrm{p}=0.8415)$. In terms of free $\mathrm{T} 4$, although bilateral TED was visually noted to have clustering around $0 \mathrm{pmol} / \mathrm{L}$ to $5 \mathrm{pmol} / \mathrm{L}$ and 10 pmol/L to $15 \mathrm{pmol} / \mathrm{L}$ (Fig. 1), unilateral TED group with mean values of $12.1 \mathrm{pmol} / \mathrm{L}$ (SD: 6.4) had similar free T4 levels with bilateral TED (8.7 $\mathrm{pmol} / \mathrm{L}$ [SD:3.1]), $(\mathrm{p}=0.1248)$. 
Table 1

Characteristics of patients by laterality of TED.

\begin{tabular}{|c|c|c|c|}
\hline Characteristics & $\begin{array}{l}\text { Unilateral }(\mathrm{n}= \\
\text { 40) }\end{array}$ & $\begin{array}{l}\text { Bilateral }(\mathrm{n}= \\
25)\end{array}$ & $\begin{array}{l}\text { p- } \\
\text { value }^{\mathrm{a}}\end{array}$ \\
\hline Age - mean (SD) & $39.1(15.2)$ & $38.7(15.6)$ & 0.9516 \\
\hline Gender (male) - no. (\%) & $12(30.0)$ & $13(52.0)$ & 0.1156 \\
\hline $\begin{array}{l}\text { Family history of thyroid disease - } \\
\text { no. (\%) }\end{array}$ & $5(12.5)$ & $5(20.0)$ & 0.4892 \\
\hline \multicolumn{4}{|l|}{ Comorbidities - no. (\%) } \\
\hline Hypertension & $9(22.5)$ & $4(16.0)$ & 0.7512 \\
\hline Diabetes mellitus & $2(5.0)$ & $3(12.0)$ & 0.3645 \\
\hline Cardiovascular disease & $2(5.0)$ & $0(0.0)$ & - \\
\hline Herniated disk & $1(2.5)$ & $0(0.0)$ & - \\
\hline Tuberculosis & $0(0.0)$ & $1(4.0)$ & - \\
\hline Myasthenia gravis & $0(0.0)$ & $1(4.0)$ & - \\
\hline Asthma & $0(0.0)$ & $1(4.0)$ & - \\
\hline Current smoker - no. (\%) & $5(12.5)$ & $2(8.0)$ & 0.6981 \\
\hline Ever smoker - no. (\%) & $5(12.5)$ & $3(12.0)$ & 0.9999 \\
\hline \multicolumn{4}{|l|}{ Current ATD use - no. (\%) } \\
\hline Methimazole & $3(7.5)$ & $8(32.0)$ & 0.0165 \\
\hline Propylthiouracil & $3(7.5)$ & $2(8.0)$ & 0.9999 \\
\hline Carbimazole & $1(2.5)$ & $0(0.0)$ & - \\
\hline
\end{tabular}

ATD: anti-thyroid medications, SD: standard deviation.

a p-value from Fisher's Exact Test for categorical variables, Mann-Whitney $U$ test for continuous variables that were not normally distributed, and $t$-test for normally distributed continuous data. Testing for normality utilized ShapiroWilk normality test.

Table 2

Signs and symptoms of TED patients.

\begin{tabular}{|c|c|c|c|}
\hline Signs and Symptoms & $\begin{array}{l}\text { Unilateral ( } \mathrm{n} \\
=40 \text { ) }\end{array}$ & $\begin{array}{l}\text { Bilateral (n } \\
=25)\end{array}$ & $\begin{array}{l}\mathrm{p}- \\
\text { value }^{\mathrm{a}}\end{array}$ \\
\hline \multicolumn{4}{|l|}{ General presentation - no. (\%) } \\
\hline Upper eyelid retraction & $26(65.0)$ & $19(76.0)$ & 0.4160 \\
\hline Exophthalmos & $26(65.0)$ & $17(68.0)$ & 0.9999 \\
\hline Exophthalmos (mm) - mean (SD) & $20.3(2.9)$ & $20.7(4.2)$ & 0.8965 \\
\hline Lid lag & $2(5.0)$ & $4(16.0)$ & 0.1938 \\
\hline Dull, deep orbital pain & $13(32.5)$ & $7(28.0)$ & 0.7868 \\
\hline Diplopia & $9(22.5)$ & $8(32.0)$ & 0.4038 \\
\hline Restricted EOMs & $15(37.5)$ & $12(48.0)$ & 0.4463 \\
\hline Lacrimation or photophobia & $3(7.5)$ & $2(8.0)$ & 0.9999 \\
\hline Blurred vision & $3(7.5)$ & $7(28.0)$ & 0.0365 \\
\hline $\begin{array}{l}\text { Decreased vision attributable to } \\
\text { optic neuropathy }\end{array}$ & $0(0.0)$ & $2(8.0)$ & - \\
\hline Lagophthalmos & $2(5.0)$ & $1(4.0)$ & 0.9999 \\
\hline Exposure symptoms & $6(15.0)$ & $7(28.0)$ & 0.2207 \\
\hline Edema & $3(7.5)$ & $2(8.0)$ & 0.9999 \\
\hline Erythema & $1(2.5)$ & $0(0.0)$ & - \\
\hline \multicolumn{4}{|l|}{ Orbital Inflammation Signs - no. (\%) } \\
\hline Lid swelling & $10(25.0)$ & $5(20.0)$ & 0.7665 \\
\hline Chemosis & $3(7.5)$ & $3(12.0)$ & 0.6681 \\
\hline Conjunctival congestion & $5(12.5)$ & $6(24.0)$ & 0.3111 \\
\hline Caruncular edema & $5(12.5)$ & $4(16.0)$ & 0.7238 \\
\hline Retrobulbar ache & $6(15.0)$ & $2(8.0)$ & 0.4711 \\
\hline Oculogyrational ache & $2(5.0)$ & $2(8.0)$ & 0.6352 \\
\hline \multicolumn{4}{|l|}{ Lid Retraction - no. (\%) } \\
\hline Upper only & $22(55.0)$ & $12(48.0)$ & 0.6182 \\
\hline Lower only & $3(7.5)$ & $6(24.0)$ & 0.0764 \\
\hline Both & $0(0.0)$ & $3(12.0)$ & - \\
\hline
\end{tabular}

SD: standard deviation.

a p-value from Fisher's Exact Test for categorical variables and Mann-Whitney $U$ test for continuous variable. Testing for normality utilized Shapiro-Wilk normality test.

In terms of severity, unilateral TED had more patients with moderate-severe TED than both mild and sight threatening TED combined (Fig. 2). Bilateral TED had a similar number of mild TED ( $\mathrm{n}=12$ [48.0\%]) and moderate-severe TED ( $n=12$ [48.0\%]); with one patient presenting with sight threatening TED (4.0\%). There was no statistically significant difference in severity by laterality of TED ( $p=0.6102)$.

Both unilateral and bilateral TED presented with predominantly inactive cases. There were similar proportions of active TED patients in unilateral TED ( $\mathrm{n}=9,22.5 \%)$ and bilateral TED groups $(\mathrm{n}=5,25 \%)$.

\section{Discussion}

Findings from the study indicate that there were more cases of unilateral TED (61.5\%) compared to bilateral TED (38.5\%). The preponderance of unilateral TED is in contrast to majority of previously published studies that investigated TED. For example, we identified one Philippine study with the local distribution of unilateral versus bilateral TED. The 2007 study found that among 58 TED patients recruited there were more patients with bilateral TED $(65.5 \%) .{ }^{17}$ In another study conducted in China it was noted that among moderate to severe TED, bilateral disease was present in $85.5 \%$ of the cases. ${ }^{18}$ An even higher difference between unilateral and bilateral TED was presented in the study from Kashkouli and colleagues; reporting that nearly 90\% were bilateral TED. ${ }^{11}$ The trend of higher bilateral TED distribution continues in studies conducted elsewhere. ${ }^{10,19-21}$

Possible explanations for the higher prevalence of unilateral TED in our study are centred around asymmetry of disease. For one, the study was conducted in a specialized ophthalmology hub. Patients may have consulted to the ophthalmology hub because of presentations of noticeable unilateral TED. ${ }^{22}$ It is possible that in bilateral disease there may be a presumption of normalcy due to symmetry of both eyes. Thus, the urgency to refer or seek consult with an ophthalmologist may be disregarded. It is in these cases as well, which leads to an illusion that the contralateral eye is not affected but was in fact subclinical. ${ }^{23}$ Kashkouli et al. discusses this at length in their paper where even ophthalmologists themselves may disregard the contralateral eye due to less proptosis, retraction, conjunctival, or lid edema versus the more prominent eye. ${ }^{11}$

Independent of the previous reasoning, another explanation could be based on the point of the disease trajectory when the patient was examined. There exists a theory suggesting a progression to bilateral TED from unilateral TED; identifying TED at the early stages consequently increases unilateral TED prevalence. ${ }^{23-26}$ Transition from unilateral to bilateral TED can take as long as seven years thus studies of longer duration may reflect varied results. ${ }^{11,23}$

Recently, a similar paper on patients from Eastern Nepal, found 82 patients presenting with TED among patients with thyroid dysfunction. ${ }^{27}$ Similar to our findings, the study had a predominance of unilateral TED (unilateral TED, $\mathrm{n}=53$; bilateral TED, $\mathrm{n}=29$ ). Although reasons behind the preponderance of unilateral TED was not discussed, the setting of the study from Eastern Nepal and that of our study have stark similarities in terms of setting; both taking place in tertiary hospitals and both are from developing countries. Therefore, an alternative explanation on the discrepancy of laterality among studies may also be that of geographic and facility-based differences. Other studies have shown differences between countries in presentation of TED and could account for varying distribution of unilateral TED versus bilateral TED as well. ${ }^{28-30}$

Stemming from the previous thought, differences between the study we conducted and the other Philippine study by Palisoc et al. could therefore be attributed to other factors. ${ }^{17}$ One difference in both studies from the Philippines is the period when the studies were done. As previously shown in literature, there appears to be a decline in severe TED in recent years, therefore suggesting an increase in early detection rate and treatment. ${ }^{30}$ In turn, it is likely that the detection rate and the point in the disease trajectory when diagnosis is made could have also influenced the changes in presentation of TED laterality between our study and the Palisoc et al. study, which was conducted more than two decades ago.

Despite the differences noted between presenting laterality of TED in our study compared to majority of the studies found, the demographic characteristics of our sample appears to be similar to other studies. Mean age for example was similar to other mean age presented in literature, including those presented in a study of Filipino TED patients. ${ }^{13,31,32}$ Mean age presented in the foregoing studies and the mean age of our 


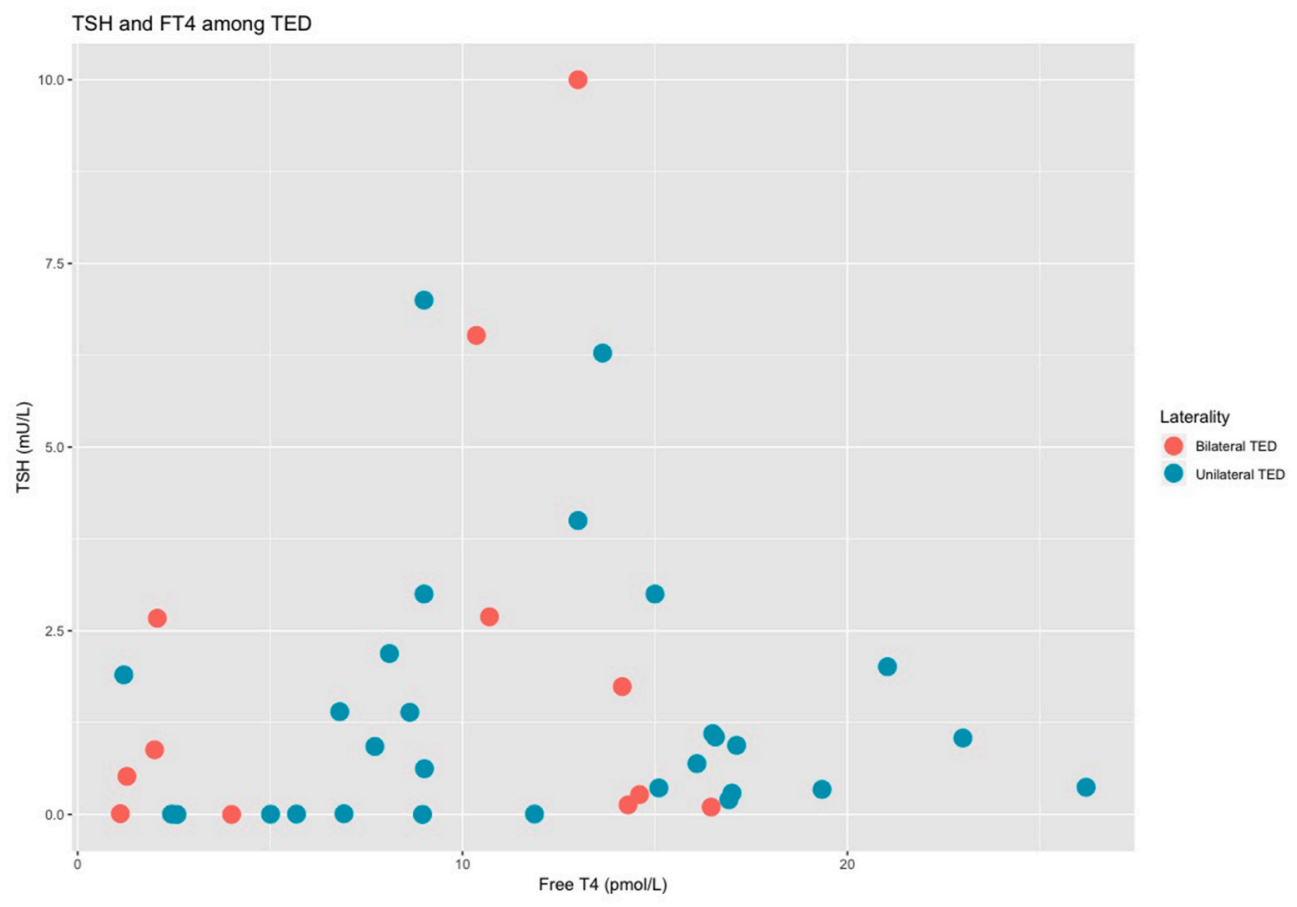

Fig. 1. Distribution of TSH and free T4 between unilateral and bilateral TED.
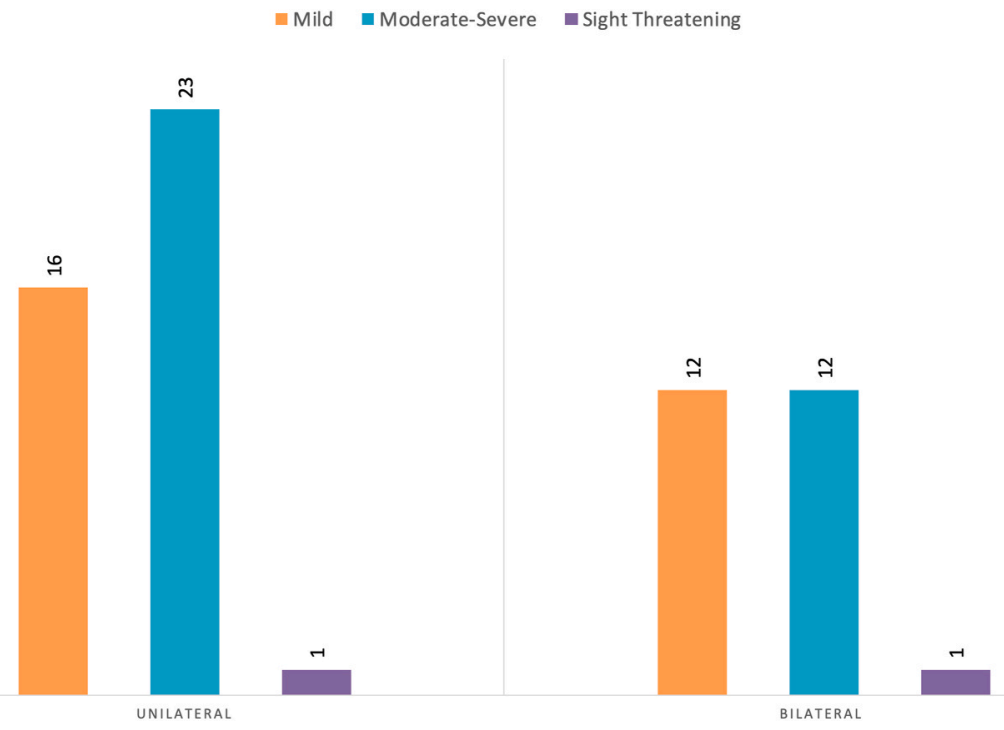

Fig. 2. TED severity between unilateral and bilateral TED.

study was all between 35 and 45 years old. With respect to laterality, our study found no difference in age between unilateral TED and bilateral TED ( $\mathrm{p}=0.9516)$. Though primary analysis of age in the study by Kashkouli et al. revealed significantly younger unilateral TED patients, their final model with age revealed no independent association between a younger age and unilateral TED. ${ }^{11}$

In terms of gender distribution, there were more females in our population, and this was most noticeable in our unilateral TED sample. However, in terms of comparing gender by laterality, differences were not statistically significant ( $\mathrm{p}=0.1556)$. Similarly, in the studies by Palisoc et al. and Kashkhouli et al. there were also more females than males and differences by laterality groupings were also not present. ${ }^{11,17}$ These findings of a higher prevalence of TED among females have also been observed outside the region. ${ }^{32-34}$

Kashkhouli and colleagues found no difference between laterality groups with regard to family history of thyroid disease and smoking history. ${ }^{11}$ Though the study by Kashkhouli et al. limited their analysis to current systemic diseases, we also found no difference in comorbidities when comparing unilateral TED and bilateral TED. We did notice, however, more bilateral TED taking methimazole $(\mathrm{p}=0.0165)$. To the best of our knowledge, there have been no other studies that have reported similar findings. Though assumptions and corresponding explanations could be made, a sample of only 11 methimazole taking patients limits the undertaking. Hence, we suggest that further investigations be made with a larger sample size.

Even with differences in geographic location and rates of thyroid disease, there appeared to be no relationship between thyroid disease and laterality of TED in our study and in the study by Kashkhouli and colleagues in Iran. ${ }^{35-37}$ Kashkhouli et al. found no difference between laterality of TED and the patient's thyroid disease. ${ }^{11}$ By using thyroid 
hormone measurements, we too did not find any difference in these parameters between laterality groups. In another study, however, Kavoussi et al., found more euthyroid patients exhibited asymmetric disease versus other groups of thyroid dysfunction. ${ }^{38}$

We found blurred vision to be associated with bilateral TED (OR = 4.80 [95\%CI: $1.11-20.76] ; \mathrm{p}=0.04$ ); a finding which we believe is secondary to the nature of the symptom being more noticeable when both eyes are involved. No other TED presentation was significant between unilateral TED and bilateral TED. Further, the similarity in presentations contributed to the outcomes of activity and severity. Our study adds to the literature, which finds activity and severity not to be different between unilateral TED and bilateral TED. ${ }^{10,11,23}$

To the best of our knowledge, we present a comparison of unilateral TED versus bilateral TED in a country where no such study has been published in 23 years. This is promising because a comparison between both studies years apart is now possible. In turn, inferences can be made on possible underpinnings, which could explain the change in presentation through time. Our study, however, is not without limitations. One, the information recorded by the attending physicians on the charts included in our study were not as comprehensive as we would have wanted. For example, we are not able to define the cause of the blurred vision based on the charting. Knowing the aetiology of the blurred vision may have uncovered other important findings and understandings. Second, as a cross-sectional study, the results presented, and the discussions derived from the findings are limited to correlations at best. Our study is not capable of defining the directionality of associations enumerated, hence no causality between variables can be made.

Nevertheless, the study was able to describe specific variables that favours a TED laterality group over another. Further analysis is warranted to understand how the variables presented here are influenced by progression of disease through time. The longitudinal study design we are suggesting, would also allow for transitions in laterality to present itself.

In conclusion, we found unilateral TED to be more prevalent than bilateral TED. Further, we found ongoing methimazole treatment and blurred vision to be strongly associated with bilateral TED compared to unilateral TED. Ultimately, our findings add to the growing evidence suggesting that the laterality of TED is not a factor in differentiating levels of activity or severity, thus showing us the very variable nature of the disease. The study encourages future longitudinal testing that aims to explore how disease progression can impact the findings realized here.

\section{Source of Funding}

The authors received no financial support for the research, authorship, and/or publication of this article.

\section{Conflict of Interest}

The authors declare no conflict of interest.

\section{References}

1 Şahlı E, Gündüz K. Thyroid-associated ophthalmopathy. Turk J Ophthalmol. 2017;47: 94-105. https://doi.org/10.4274/tjo.80688.

2 McAlinden C. An overview of thyroid eye disease. Eye Vis (Lond). 2014;1:9. https:// doi.org/10.1186/s40662-014-0009-8.

3 Belliveau MJ, Jordan DR. Thyroid eye disease. CMAJ (Can Med Assoc J). 2013;185: 797. https://doi.org/10.1503/cmaj.121815.

4 American Thyroid Association and American Association of Clinical Endocrinologists Taskforce on Hyperthyroidism and Other Causes of Thyrotoxicosis, Bahn RS, Burch HB, et al. Hyperthyroidism and other causes of thyrotoxicosis: management guidelines of the American thyroid association and American association of clinical endocrinologists. Thyroid. 2011;21:593-646. https://doi.org/10.1089/ thy. 2010.0417 .

5 Tramunt B, Imbert P, Grunenwald S, Boutault F, Caron P. Sight-threatening Graves' orbitopathy: twenty years' experience of a multidisciplinary thyroid-eye outpatient clinic. Clin Endocrinol. 2019;90:208-213. https://doi.org/10.1111/cen.13880.
6 Wall JR, Lahooti H. Pathogenesis of thyroid eye disease-does autoimmunity against the TSH receptor explain all cases? Endokrynol Pol. 2010;61:222-227.

7 Lehmann GM, Feldon SE, Smith TJ, Phipps RP. Immune mechanisms in thyroid eye disease. Thyroid. 2008;18:959-965. https://doi.org/10.1089/thy.2007.0407.

8 Prabhakar BS, Bahn RS, Smith TJ. Current perspective on the pathogenesis of Graves' disease and ophthalmopathy. Endocr Rev. 2003;24:802-835.

9 Kalmann R, Mourits MP. Late recurrence of unilateral Graves' orbitopathy on the contralateral side. Am J Opthalmol. 2002;5:7727-7729.

10 Wiersinga WM, Smit T, Van der Graag R, Mourits M, Korneef L. Ophthalmic Res. 1989;21:73-82.

11 Kashkouli MB, Kaghazkanani R, Heidari I, et al. Bilateral versus unilateral thyroid eye disease. Indian J Ophthalmol. 2011;59:363.

12 Weiler DL. Thyroid eye disease: a review. Clin Exp Optom. 2017;100:20-25.

13 Bartley GB, Gorman CA. Diagnostic criteria for Graves' ophthalmopathy. Am J Ophthalmol. 1995 Jun;119(6):792-795.

14 Lat AM, Jauculan MC, Sanchez CA, et al. Risk factors associated with the activity and severity of Graves' ophthalmopathy among patients at the University of the Philippines Manila-Philippine General Hospital. Journal of the ASEAN Federation of Endocrine Societies. 2017;32:151.

15 Mourits MP, Koornneef L, Wiersinga WM, Prummel MF, Berghout A, van der Gaag R. Clinical criteria for the assessment of disease activity in Graves' ophthalmopathy: a novel approach. BJO (Br J Ophthalmol). 1989;73:639-644.

16 Barrio-Barrio J, Sabater AL, Bonet-Farriol E, Velázquez-Villoria Á, Galofré JC. Graves' ophthalmopathy: VISA versus EUGOGO classification, assessment, and management. J Ophthalmol. 2015:249125. https://doi.org/10.1155/2015/249125, 2015.

17 Palisoc ED, Morabe ES, Pagkatipunan PMN. Prevalence of Graves ophthalmology among patients with thyroid disease. Philippine J Ophthalmol. 2010;35:32-35.

$18 \mathrm{Li} \mathrm{Q}$, Ye H, Ding Y, et al. Clinical characteristics of moderate-to-severe thyroid associated ophthalmopathy in 354 Chinese cases. PLoS One. 2017;12, e0176064. https://doi.org/10.1371/journal.pone.0176064.

19 Lim NCS, Sundar G, Amrith S, Lee KO. Thyroid eye disease: a Southeast Asian experience. Br J Ophthalmogy. 2014:1-7. https://doi.org/10.1136/bjophthalmol 2014-305649, 0

20 Ackuaku-Dogbe EM, Akpalu J, Abaidoo B. Epidemiology and clinical features of thyroid-associated orbitopathy in Accra. Middle East Afr J Ophthalmol. 2017;24: 183-189.

21 Wiersinga WM. Graves' orbitopathy: management of difficult cases. Indian J Endocrinol Metabol. 2012;16:S150-S152. https://doi.org/10.4103/22308210.104026.

22 Perros P, Neoh C, Dickinson J. Thyroid eye disease. BMJ. 2009;338:b560. https:// doi.org/10.1136/bmj.b560.

23 Strianese D, Piscopo R, Elefante A, et al. Unilateral proptosis in thyroid eye disease with subsequent contralateral involvement: retrospective follow-up study. BMC Ophthalmol. 2013;13:21. https://doi.org/10.1186/1471-2415-13-21.

24 Daumerie Ch, Duprez Th, Boschi A. Long-term multidisciplinary follow-up of unilateral thyroid-associated orbitopathy. Eur J Intern Med. 2008;19:531-536.

25 Perrild H, Feldt-Rusmussen U, Bech K, Ahlgren P, Hansen JM. The differential diagnosis problems in unilateral euthyroid Graves' ophthalmopathy. Acta Endocrinol. 1984;106:471.

26 Kashkouli MB. Beyond a typical thyroid eye disease. Middle East Afr J Ophthalmol. 2013;20:185-186. https://doi.org/10.4103/0974-9233.114787.

27 Lavaju P, Badhu BP, Maskey R. Pattern of ocular manifestations in patients with thyroid disease presenting in Eastern Nepal. Thyroid Res Pract. 2019;16:20-25.

28 Bartley GB. The epidemiologic characteristics and clinical course of ophthalmopathy associated with autoimmune thyroid disease in Olmsted county, Minnesota. Trans Am Ophthalmol Soc. 1994;92:477-588.

29 Yang DD, Gonzalez MO, Durairaj VD. Medical management of thyroid eye disease. Saudi J Ophthalmol. 2011;25:3-13. https://doi.org/10.1016/j.sjopt.2010.10.001.

30 Chng CL, Seah SL, Khoo DH. Ethnic differences in the clinical presentation of Graves ophthalmopathy. Best Pract Res Clin Endocrinol Metabol. 2012;26:10.

31 Laurberg PBD, Bülow Pedersen I, Andersen S, Carlé A. Incidence and clinical presentation of moderate to severe graves' orbitopathy in a Danish population before and after iodine fortification of salt. J Clin Endocrinol Metab. 2012;97:8.

32 Kashkouli MB, Jam S, Sabzvari D, et al. Thyroid-associated ophthalmopathy in Iranian patients. Acta Med Iran. 2011;49:612-618.

33 Kendler DL, Lippa J, Rootman J. The initial clinical characteristics of Graves orbitopathy vary with age and sex. Arch Ophthalmol. 1993;111:197-201.

34 Perros PCA, Matthews JN, Kendall-Taylor P. Age and gender influence the severity of thyroid-associated ophthalmopathy: a study of 101 patients attending a combined thyroid-eye clinic. Clin Endocrinol. 1993;38:6.

35 Abraham-Nordling MBK, Törring O, Lantz M, et al. Incidence of hyperthyroidism in Sweden. Eur J Endocrinol. 2011;165:7.

36 Taylor PN, Albrecht D, Scholz A, et al. Global epidemiology of hyperthyroidism and hypothyroidism. Nat Rev Endocrinol. 2018;14:301.

37 Carlos-Raboca J, Jimeno CA, Kho SA, et al. The Philippine Thyroid Diseases Study (PhilTiDes 1): prevalence of thyroid disorders among adults in the Philippines. Journal of the ASEAN Federation of Endocrine Societies. 2014;27:27.

38 Kavoussi SC, Giacometti JN, Servat J, Levin F. The relationship between sex and symmetry in thyroid eye disease. Clin Ophthalmol. 2014;8:1295. https://doi.org/ 10.2147/OPTH.S61041. 